\title{
Partial FOV Center Imaging (PCl): A Robust $X$-Space Image Reconstruction for Magnetic Particle Imaging
}

\author{
Semih Kurt $^{\circledR}$, Yavuz Muslu ${ }^{\circledR}$, and Emine Ulku Saritas ${ }^{(}$
}

\begin{abstract}
Magnetic Particle Imaging (MPI) is an emerging medical imaging modality that images the spatial distribution of superparamagnetic iron oxide (SPIO) nanoparticles using their nonlinear response to applied magnetic fields. In standard X-space approach to MPI, the image is reconstructed by gridding the speed-compensated nanoparticle signal to the instantaneous position of the field free point (FFP). However, due to safety limits on the drive field, the field-of-view (FOV) needs to be covered by multiple relatively small partial field-of-views (pFOVs). The image of the entire FOV is then pieced together from individually processed pFOVs. These processing steps can be sensitive to non-ideal signal conditions such as harmonic interference, noise, and relaxation effects. In this work, we propose a robust $x$-space reconstruction technique, Partial FOV Center Imaging (PCI), with substantially simplified pFOV processing. $\mathrm{PCl}$ first forms a raw image of the entire FOV by mapping MPI signal directly to pFOV center locations. The corresponding MPI image is then obtained by deconvolving this raw image by a compact kernel, whose fully-known shape solely depends on the pFOV size. We analyze the performance of the proposed reconstruction via extensive simulations, as well as imaging experiments on our in-house FFP MPI scanner. The results show that $\mathrm{PCl}$ offers a trade-off between noise robustness and interference robustness,
\end{abstract}

Manuscript received November 15, 2019; revised April 12, 2020; accepted May 8, 2020. Date of publication May 18, 2020; date of current version October 28,2020 . This work was supported by the Scientific and Technological Research Council of Turkey under Grant TUBITAK 115E677. (Corresponding author: Semih Kurt.)

Semih Kurt is with the Department of Electrical and Electronics Engineering, Bilkent University, 06800 Ankara, Turkey, and also with the National Magnetic Resonance Research Center (UMRAM), Bilkent University, 06800 Ankara, Turkey (e-mail: kurt@ee.bilkent.edu.tr).

Yavuz Muslu was with the Department of Electrical and Electronics Engineering, Bilkent University, 06800 Ankara, Turkey, and also with the National Magnetic Resonance Research Center (UMRAM), Bilkent University, 06800 Ankara, Turkey. He is now with the Department of Biomedical Engineering, University of Wisconsin-Madison, Madison, WI 53706 USA, and also with the Department of Radiology, University of Wisconsin-Madison, Madison, WI 53706 USA (e-mail: ymuslu@wisc.edu).

Emine Ulku Saritas is with the Department of Electrical and Electronics Engineering, Bilkent University, 06800 Ankara, Turkey, also with the National Magnetic Resonance Research Center (UMRAM), Bilkent University, 06800 Ankara, Turkey, and also with the Neuroscience Program, Sabuncu Brain Research Center, Bilkent University, 06800 Ankara, Turkey (e-mail: saritas@ee.bilkent.edu.tr).

Color versions of one or more of the figures in this article are available online at http://ieeexplore.ieee.org.

Digital Object Identifier 10.1109/TMI.2020.2995410 outperforming standard $\mathrm{x}$-space reconstruction in terms of both robustness against non-ideal signal conditions and image quality.

Index Terms-Magnetic particle imaging, image reconstruction, harmonic interference robustness, noise robustness, deconvolution.

\section{INTRODUCTION}

$\mathbf{M}$ AGNETIC Particle Imaging (MPI) leverages the nonlinear magnetization response of superparamagnetic iron oxide (SPIO) nanoparticles, to generate an image of their spatial distribution with high resolution, contrast, and sensitivity [1]-[6]. Three different magnetic fields are utilized to obtain the MPI signal. A static selection field with a strong gradient creates a field free point (FFP). A sinusodial drive field excites the nanoparticles in the vicinity of the FFP, effectively scanning a field-of-view (FOV) via moving the FFP. However, safety limits restrict the size of the FOV that can be scanned by the drive field alone to a few $\mathrm{cm}$ or less [7], [8]. The small FOV covered by the drive field is called a partial FOV (pFOV) [9] or a patch [10]-[12]. Then, to cover larger FOVs, low-frequency focus fields are employed [13]. Due to the limits on the slew rates of the focus fields [8], [14], a realistic scan trajectory may consist of numerous highly-overlapping small pFOVs. In $\mathrm{x}$-space reconstruction, these pFOVs are first individually processed by gridding the speed-compensated signal to the trajectory of the FFP, and then combined to form the image of the entire FOV [9], [15]-[19]. The resulting MPI image shows the spatial distribution of the nanoparticles blurred by the point spread function (PSF) of the imaging system.

One of the challenges in MPI is the direct feedthrough signal caused by the simultaneous excitation and reception, as it can be many orders of magnitude larger than the nanoparticle signal [15], [16]. To keep the direct feedthrough contained to the fundamental harmonic, the drive field is typically chosen as a pure sinusoid. Then, a gradiometric receive coil [20]-[22], active/passive compensation [23], and/or analog/digital filtering can be utilized to counteract the effects of the direct feedthrough. During image reconstruction with the $\mathrm{x}$-space approach, the fundamental harmonic lost due to filtering is recovered via enforcing smoothness and non-negativity on 
the reconstructed image [9], [24]. A 3D extension of this approach was also formulated as a convex optimization problem, ensuring the consistency of the recovered information across pFOVs [17].

If the received signal also contains higher harmonics due to system non-idealities and/or interferences, however, simple filtering no longer suffices. In practice, therefore, a background measurement is subtracted from the received signal to cancel out potential higher harmonic interferences. If the interference level is comparable to the nanoparticle signal, or if there is a drift in the system, this background cancellation may not work as desired. While the aforementioned $\mathrm{x}$-space reconstruction techniques enforced consistency, smoothness and non-negativity, a detailed analysis on the detrimental effects of higher harmonic interference on the quality of the reconstructed MPI image has not yet been presented.

In this work, we present a robust $\mathrm{x}$-space image reconstruction technique called "pFOV center imaging" (PCI), which features substantially simplified pFOV processing and increased robustness against harmonic interferences. The proposed technique first forms a raw image of the entire FOV by mapping the MPI signal directly to the pFOV center locations. Then, this raw image is deconvolved by a fully known, compact kernel to obtain the corresponding MPI image. Importantly, the shape of this kernel solely depends on the pFOV size, and is independent of the other scanning parameters or the nanoparticle type. We analyze the performance of the proposed method at different signal-to-noise ratio (SNR) and harmonic interference levels, demonstrating a trade-off between noise robustness and harmonic interference robustness. With extensive simulations, as well as imaging experiments on a FFP scanner, we show that PCI outperforms standard $\mathrm{x}$-space reconstruction in terms of image quality, noise robustness, and interference robustness.

\section{THEORY}

For a trajectory that contains a 1D drive field superimposed with a slowly varying focus field, the pFOV centers are closely spaced and the FFP speed is dominated by the drive field. For such a trajectory, the time-domain MPI signal, $s(t)$, can be written as [15], [16]:

$$
s(t)=\alpha \dot{x}_{s}(t) \hat{\rho}\left(x_{s}(t)\right)
$$

where

$$
\hat{\rho}\left(x_{s}(t)\right)=\left.\rho(x) * h(x)\right|_{x=x_{S}(t)}
$$

Here, $x_{s}(t)$ is the instantaneous FFP position, $\dot{x}_{s}(t)$ is the instantaneous FFP speed, $\rho(x)$ is the particle distribution, $h(x)$ is the PSF, and $\hat{\rho}(x)$ is the PSF-blurred "ideal" MPI image. In addition, $\alpha$ is a constant that depends on the selection field gradient, the nanoparticle type, the magnetic moment of the nanoparticle, and the sensitivity of the receive coil [15], [16]. For the following derivations, we ignore the nanoparticle relaxation effects on the signal.

Due to direct feedthrough filtering, the MPI signal loses its fundamental harmonic component. It has been shown that the contribution of the lost first harmonic for each pFOV is a DC term [9], [25]. In standard $\mathrm{x}$-space reconstruction, DC terms are recovered via pFOV stitching by enforcing non-negativity and continuity constraints on the reconstructed image [9].

\section{A. Partial FOV Center Imaging (PCI)}

Let $x_{0 j}$ be the center position of the $j^{\text {th }} \mathrm{pFOV}$ and $t_{0 j}$ be the time instant when the FFP is at $x_{0 j}$, i.e.,

$$
\left.x_{s}(t)\right|_{t=t_{0 j}}=x_{0 j}, \quad \text { for } j=1, \ldots, N
$$

where $N$ is the total number of pFOVs. We propose to sample $s(t)$ at the centers of pFOVs to get a raw image $\hat{\rho}_{0}(x)$ such that

$$
\begin{aligned}
\hat{\rho}_{0}\left(x_{0 j}\right) & =\left.s(t)\right|_{t=t_{0 j}} \\
& =\alpha \dot{x}_{s}\left(t_{0 j}\right) \hat{\rho}\left(x_{0 j}\right) \\
& =\beta_{0} \hat{\rho}\left(x_{0 j}\right), \quad \text { for } j=1, \ldots, N
\end{aligned}
$$

For the trajectories considered here, because the FFP speed is dominated by the drive field, it can be considered as identical at the centers of pFOVs. Therefore, $\beta_{0}=\alpha \dot{x}_{s}\left(t_{0 j}\right)$ is a constant for $j=1, \ldots, N$.

In practice, due to direct feedthrough filtering, $\hat{\rho}_{0}(x)$ is devoid of the contribution of the first harmonic. We have previously shown that the lost DC term for the $j^{\text {th }} \mathrm{pFOV}$ can be represented by a convolution as [26]:

$$
\hat{\rho}_{d c}\left(x_{0 j}\right)=\left.\frac{4}{\pi W}\left(\hat{\rho}(x) * \sqrt{1-\left(\frac{2 x}{W}\right)^{2}}\right)\right|_{x=x_{0 j}}
$$

where $W$ is the extent of each pFOV. Note that while this expression was originally derived for stepped focus fields, it also applies when the focus fields are slowly varying. Taking into account the lost DC term and using Eqs. 4c and 5, the raw image at $x_{0 j}$ can be expressed as

$$
\begin{aligned}
\tilde{\rho}_{0}\left(x_{0 j}\right) & =\beta_{0}\left(\hat{\rho}\left(x_{0 j}\right)-\hat{\rho}_{d c}\left(x_{0 j}\right)\right) \\
& =\left.\beta_{0}\left(\hat{\rho}(x) *\left(\delta(x)-\frac{4}{\pi W} \sqrt{1-\left(\frac{2 x}{W}\right)^{2}}\right)\right)\right|_{x=x_{0 j}}
\end{aligned}
$$

valid for all $j=1, \ldots, N$. Hence, the raw image $\tilde{\rho}_{0}(x)$ can be written as the ideal image convolved with a kernel, i.e.,

$$
\tilde{\rho}_{0}(x)=\hat{\rho}(x) * h_{0}(x)
$$

where

$$
h_{0}(x)=\beta_{0}\left(\delta(x)-\frac{4}{\pi W} \sqrt{1-\left(\frac{2 x}{W}\right)^{2}}\right)
$$

Note that $h_{0}(x)$ is a compact kernel with full-width $W$, and is fully known upto a scaling factor $\beta_{0}$. Importantly, the shape of the kernel does not depend on the nanoparticle type. Next, we can deconvolve $\tilde{\rho}_{0}(x)$ by the known kernel $h_{0}(x)$ to obtain $\hat{\rho}(x)$, i.e.,

$$
\hat{\rho}(x)=\tilde{\rho}_{0}(x) *^{-1} h_{0}(x)
$$


(a) MPI Signals for Different pFOVs

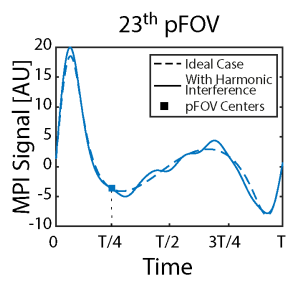

(b) pFOV Images

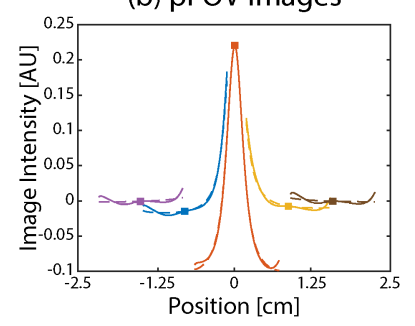

Fig. 1. Effects of harmonic interference on the MPI signal and image reconstruction. (a) The signals after direct feedthrough filtering and (b) the reconstructed images from selected pFOVs with and without harmonic interference. The centers of pFOVs are particularly robust against interferences, while the edges can exhibit large deviations. (c) The reconstructed MPI images for $\mathrm{PCl}$ vs. standard $\mathrm{x}$-space reconstruction. Standard $\mathrm{x}$-space reconstruction exhibits an accumulated error toward one end of the image, while PCl image is free from such artifacts. Here, the ideal case refers to the convolution of the nanoparticle distribution with the imaging PSF. These simulations were performed for a point source placed at the origin, with $2.4 \mathrm{~T} / \mathrm{m} / \mu_{0}$ gradient, $10 \mathrm{mT}$ drive field at $9.7 \mathrm{kHz}, 10 \mathrm{~T} / \mathrm{s}$ slew-rate.

Here, $*^{-1}$ denotes the deconvolution operation. We refer to this technique as pFOV center imaging (PCI).

Because PCI utilizes only the signals at pFOV centers, it is particularly robust against harmonic interference effects. This robustness is directly related to the MPI harmonic image basis set, which is composed of Chebyshev polynomials of the second kind [9], [25]. Independent of the harmonic number, each image basis varies relatively slowly near the pFOV centers when compared to locations towards the edges. In return, the pFOV centers are particularly robust against harmonic interference effects, whereas the edges are sensitive to such interferences. A pictorial depiction of this effect is demonstrated in Fig. 1, where the MPI signal and corresponding images from different $\mathrm{pFOV}$ s are shown with and without harmonic interference (see Section III-B for details on these simulations). As seen Fig. 1a-b, the MPI signals and pFOV images corresponding to the $\mathrm{pFOV}$ centers are approximately the same with and without interference, whereas other positions can exhibit large deviations. These deviations make it more difficult to estimate the lost DC term, causing standard $\mathrm{x}$-space reconstruction to exhibit an accumulated error toward one end of the image, as shown in Fig. 1c. On the other hand, the MPI image reconstructed using PCI does not exhibit such an artifact.

\section{B. Lumped PCI}

Since PCI uses only a small portion of the received signal, it may be affected by noise. To increase robustness against noise, PCI can be modified to use all the received signal. Let $x_{k j}$ be the $k^{\text {th }}$ sampling position in the $j^{\text {th }} \mathrm{pFOV}$ and $t_{k j}$ be the time instant when the FFP is at $x_{k j}$, i.e.,

$$
\left.x_{s}(t)\right|_{t=t_{k j}}=x_{k j}, \quad \text { for } j=1, \ldots, N, k=-K, \ldots, K
$$

where $2 K+1$ is the number of samples in one half drive field cycle. A raw image $\hat{\rho}_{k}(x)$ can be constructed by sampling $s(t)$ at positions $x_{k j}$, but assigning these samples to center positions $x_{0 j}$ :

$$
\begin{aligned}
\hat{\rho}_{k}\left(x_{0 j}\right) & =\alpha \dot{x}_{s}\left(t_{k j}\right) \hat{\rho}\left(x_{k j}\right) \\
& =\beta_{k} \hat{\rho}\left(x_{k j}\right)
\end{aligned}
$$

Note that the FFP speed is identical at the $k^{\text {th }}$ sampling positions of pFOVs. Therefore $\beta_{k}=\alpha \dot{x}_{s}\left(t_{k j}\right)$ is a constant for $j=1, \ldots, N$.

Direct feedthrough filtering causes the same DC loss at all positions in a given FOV [9], [26]. Hence, similar to Eq. 6a, the raw image with lost DC term can be expressed as

$$
\tilde{\rho}_{k}\left(x_{0 j}\right)=\beta_{k}\left(\hat{\rho}\left(x_{k j}\right)-\hat{\rho}_{d c}\left(x_{0 j}\right)\right)
$$

valid for all $j=1, \ldots, N$. By comparing Eqs. 6a and 12,

$$
\begin{aligned}
\tilde{\rho}_{k}\left(x_{0 j}\right)= & \beta_{k}\left(\left.\hat{\rho}(x) * \delta\left(x-\left(x_{0 j}-x_{k j}\right)\right)\right|_{x=x_{0 j}}-\hat{\rho}_{d c}\left(x_{0 j}\right)\right) \\
= & \beta_{k}\left(\hat { \rho } ( x ) * \left(\delta\left(x-\left(x_{0 j}-x_{k j}\right)\right)\right.\right. \\
& \left.\left.-\frac{4}{\pi W} \sqrt{1-\left(\frac{2 x}{W}\right)^{2}}\right)\right)\left.\right|_{x=x_{0 j}}
\end{aligned}
$$

We can rewrite the $k^{t h}$ raw image $\tilde{\rho}_{k}(x)$ in a simplified form:

$$
\tilde{\rho}_{k}(x)=\hat{\rho}(x) * h_{k}(x)
$$

where

$$
h_{k}(x)=\beta_{k}\left(\delta\left(x-\left(x_{0 j}-x_{k j}\right)\right)-\frac{4}{\pi W} \sqrt{1-\left(\frac{2 x}{W}\right)^{2}}\right)
$$

Then, one can reconstruct $\hat{\rho}(x)$ via deconvolving $\tilde{\rho}_{k}(x)$ by $h_{k}(x)$ :

$$
\hat{\rho}(x)=\tilde{\rho}_{k}(x) *^{-1} h_{k}(x)
$$

Eq. 14 shows that sampling $s(t)$ at different positions in pFOVs can create different raw images, and one can obtain $\hat{\rho}(x)$ from any of these raw images. Note that among all $\tilde{\rho}_{k}(x)$, the one with the highest SNR is $\tilde{\rho}_{0}(x)$, as the FFP speed is maximum when passing through the pFOV center. To boost the SNR and use the entire signal, we can sum all $\tilde{\rho}_{k}(x)$ to get a raw lumped image, i.e.,

$$
\begin{aligned}
\tilde{\rho}_{\text {lum }}(x) & =\sum_{k=-K}^{K} \tilde{\rho}_{k}(x) \\
& =\hat{\rho}(x) * h_{\text {lum }}(x)
\end{aligned}
$$


(a) $\hat{\rho}(x)$ for different k's

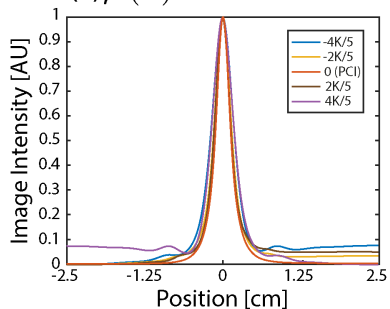

(b) Lumped-PCI vs. X-space

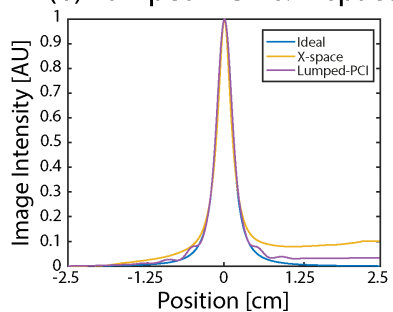

Fig. 2. Effects of sampling position $k$ on the MPI image reconstruction under harmonic interference. (a) The reconstructed images using Eq. 16 for different $k$ s, where $k=0$ image corresponds to $\mathrm{PCl}$ (i.e., using the centers of the pFOVs). As $|k|$ gets larger (i.e., using sampling positions near pFOV edges), the accumulated error toward one end of the image gets stronger. (b) The reconstructed images for Lumped-PCl vs. standard $\mathrm{x}$-space reconstruction. Lumped-PCI provides interference robustness by effectively averaging out the negative/positive deviations from different $k$ 's, yielding only slight ripple-like artifacts.

where

$$
h_{\text {lum }}(x)=\sum_{k=-K}^{K} h_{k}(x)
$$

Eq. 18 follows from the linearity of the convolution operation. Once again, we can reconstruct $\hat{\rho}(x)$ via deconvolution:

$$
\hat{\rho}(x)=\tilde{\rho}_{\text {lum }}(x) *^{-1} h_{\text {lum }}(x)
$$

We refer to this extension of the method as Lumped-PCI.

While Lumped-PCI boosts SNR and improves noise robustness, it is slightly more sensitive against harmonic interferences when compared to PCI due to the usage of the pFOV edges. As seen in Fig. 2a, the reconstructed images using Eq. 16 for larger $|k|$ (i.e., using sampling positions near pFOV edges) exhibit accumulated error toward one end of the image. In contrast, Lumped-PCI image in Fig. $2 b$ shows only slight ripple-like artifacts. As Lumped-PCI sums the raw images from the entire $\mathrm{pFOV}$, it effectively averages out the negative/positive deviations from the ideal values from different $k$ 's. Hence, Lumped-PCI still displays increased reconstruction fidelity and interference robustness when compared to standard $\mathrm{x}$-space reconstruction.

\section{METHODS}

\section{A. Simulations}

MPI simulations were carried out using a custom toolbox developed in MATLAB (Mathworks, Natick, MA). Simulation parameters were chosen to match the parameters of our in-house FFP MPI scanner (Fig. 3a). Accordingly, the selection field gradients were $(-4.8,2.4,2.4) \mathrm{T} / \mathrm{m} / \mu_{0}$ in $(\mathrm{x}, \mathrm{y}, \mathrm{z})$ directions. $10 \mathrm{mT}$ drive field at $9.7 \mathrm{kHz}$ along the z-direction was simulated together with a focus field that creates $1 \mathrm{~T} / \mathrm{s}$ slew rate in the z-direction. We used a $5 \times 5 \mathrm{~cm}^{2}$ vasculature phantom and assumed $25 \mathrm{~nm}$ nanoparticle diameter. The pFOV size was $8.33 \mathrm{~mm}$ and a 2D FOV of $5 \times 5 \mathrm{~cm}^{2}$ was scanned using a linear trajectory, similar to the one shown in Fig. $3 \mathrm{~b}$. The entire FOV was scanned in 51 lines. To match the conditions of the imaging experiments, the simulated MPI signal was sampled at $2 \mathrm{MS} / \mathrm{s}$.

\section{B. Noise and Harmonic Interference Robustness Analysis}

To analyze the robustness of the proposed method, we simulated noise, harmonic interference, and relaxation effects on MPI signal. For noise analysis, white Gaussian noise was added to the time-domain MPI signal at 10 different noise levels, with signal-to-noise ratio (SNR) varying between 5-50 dB. SNR was defined using the peak signal amplitude as follows:

$$
S N R=20 \log _{10}\left(\frac{\max _{t}|s(t)|}{\sigma}\right)
$$

Here, $\sigma$ denotes the standard deviation of noise, and $s(t)$ is the MPI signal after direct feedthrough filtering.

For harmonic interference analysis, harmonic interference was added to the spectrum of $s(t)$. When the drive field is applied alone, the spectrum of the MPI signal contains only the harmonics of the fundamental frequency, $f_{0}$ [15]. However, for the linear scan trajectory used in this work, the harmonics spread to very narrow nearby bands [27]. Let $S(f)$ denote the Fourier transform of $s(t)$ and $S_{n}(f)$ denote the $n^{t h}$ harmonic band, i.e.,

$$
S_{n}(f)= \begin{cases}S(f), & \left(n-\frac{1}{2}\right) f_{0}<f<\left(n+\frac{1}{2}\right) f_{0} \\ 0, & \text { otherwise }\end{cases}
$$

The magnitude of the interference added to the $n^{\text {th }}$ harmonic (i.e., at $f=n f_{0}$ ) was uniformly distributed between 0 and $\gamma_{n}$, whereas the phase of it was uniformly distributed between 0 and $2 \pi$. To assess the strength of the MPI signal against harmonic interference, we used the signalto-interference ratio (SIR) metric. Based on our experimental observations, harmonic interference was simulated so that each harmonic band had the same SIR level, i.e.,

$$
S I R=20 \log _{10}\left(\frac{\max _{f}\left|S_{n}(f)\right|}{\gamma_{n}}\right)
$$

Hence, as the magnitude spectrum in MPI decayed at higher harmonics, the interference followed the same trend. For simulations, 6 different SIR levels between 4-24 dB were tested.

First, we simulated noise and harmonic interference effects separately. Then, we incorporated both effects simultaneously with SNR ranging between 5-50 dB and SIR ranging between 4-24 dB. Monte Carlo simulations were performed via repeating each case 50 times. Next, to incorporate the effects of relaxation, we utilized a realistic time constant of $\tau=3 \mu \mathrm{s}$ [28], [29], using the model provided in [30]. For this analysis, SNR was fixed at $30 \mathrm{~dB}$ and SIR at $8 \mathrm{~dB}$.

\section{Imaging Experiments}

Imaging experiments were performed on in-house FFP MPI scanner (Fig. 3) [31]. The selection field of this scanner was generated by two permanent magnets with $7-\mathrm{cm}$ diameter and $2-\mathrm{cm}$ thickness, placed at $8-\mathrm{cm}$ separation. The resulting selection field gradients were $(-4.8,2.4,2.4) \mathrm{T} / \mathrm{m} / \mu_{0}$ in $(\mathrm{x}, \mathrm{y}, \mathrm{z})$ 
(a) In-house FFP MPI Scanner

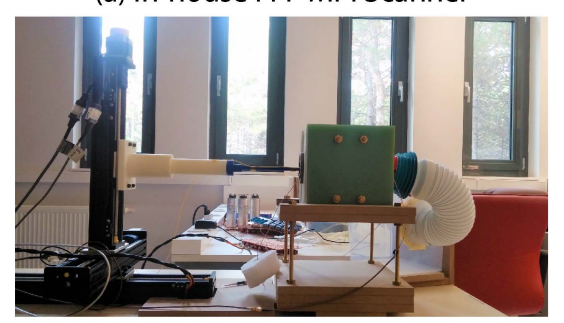

(b) Linear Scan Trajectory

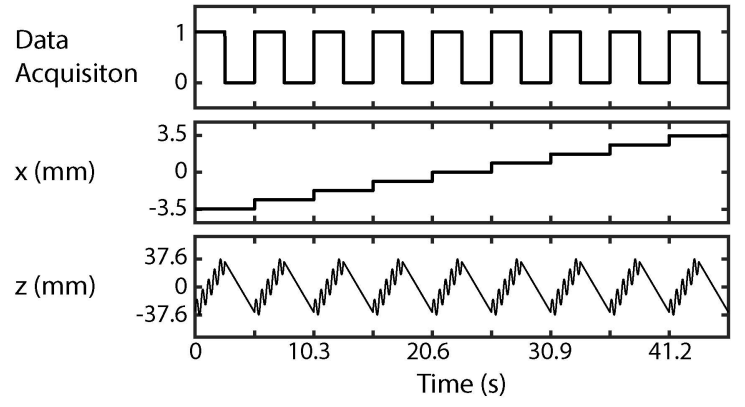

Fig. 3. An overview of our in-house FFP MPI scanner and the linear scan trajectory used in the imaging experiments. (a) This scanner features $(-4.8,2.4,2.4) \mathrm{T} / \mathrm{m} / \mu_{0}$ selection field gradients in $(x, y, z)$ directions, with a maximum FOV of $1 \times 1 \times 10 \mathrm{~cm}^{3}$. (b) The linear scan trajectory had $0.7 \times 7.52 \mathrm{~cm}^{2} \mathrm{FOV}$ in $\mathrm{x}-\mathrm{z}$ plane, $0.07 \mathrm{~T} / \mathrm{s}$ slew rate along $\mathrm{z}$-direction using continuous robotic arm motion, with $23.2 \mathrm{sec}$ active scan time. This figure shows a simplified version of the actual trajectory: instead of the drive field at $9.7 \mathrm{kHz}$ and $10 \mathrm{mT}$-peak, a representative drive field at a much lower frequency is plotted.

directions. The drive field coil had a $1.5 \mathrm{mT} / \mathrm{A}$ sensitivity, with $95 \%$ homogeneity in a $4.5-\mathrm{cm}$ long region, and was built using 3 layers of Litz wire with 80 turns. For the receive coil, a three-section gradiometer type coil with 34 and 17.5 windings for the main section and side sections was utilized [20]. The drive and receive coils were positioned coaxially, and placed inside a cylindrical copper shield with $1-\mathrm{cm}$ thickness at the center of the magnet configuration. The maximum FOV of this FFP MPI scanner is $1 \times 1 \times 10 \mathrm{~cm}^{3}$.

In imaging experiments, $0.7 \times 7.52 \mathrm{~cm}^{2} \mathrm{FOV}$ in $\mathrm{x}-\mathrm{z}$ plane was scanned. To cover this FOV, the linear trajectory shown in Fig. 3b was utilized. The drive field was at $9.7 \mathrm{kHz}$ and $10 \mathrm{mT}$-peak along the z-direction, resulting in a $8.33 \mathrm{~mm}$ pFOV length. Instead of a focus field, a three-axis robotic arm (Motor-Driven Velmex BiSlide, Model: MN10-0100-E0121) was used to move the phantom continuously along the $z$ direction, and stepwise along the $\mathrm{x}$-direction. The maximum slew rate for the continuous motion was $0.07 \mathrm{~T} / \mathrm{s}$ due to the speed limitation of this mechanical system. The entire FOV was scanned in 9 lines, with an active scan time of $23.2 \mathrm{sec}$.

\section{Imaging Phantoms}

Two different imaging phantoms were prepared to demonstrate the performance of the proposed method. For the first phantom, two 3-mm vials were filled with Perimag (Micromod $\mathrm{GmbH}$, Germany) nanoparticles with a diluted concentration of $5 \mathrm{mg} \mathrm{Fe} / \mathrm{mL}$. Vials were separated by $9-\mathrm{mm}$ distance along the $\mathrm{z}$-direction.
To show that the proposed method can successfully handle different nanoparticle types, a second phantom was prepared. For this phantom, three 2-mm inner diameter vials were filled with Nanomag-MIP (Micromod GmbH, Germany) nanoparticles with a diluted concentration of $1.43 \mathrm{mg}$ Fe/mL, Vivotrax (Magnetic Insight Inc., USA) with an undiluted concentration of $5.5 \mathrm{mg} \mathrm{Fe} / \mathrm{mL}$, and a homogeneous mixture of the two. The vials were separated by $15-\mathrm{mm}$ distances along the $\mathrm{z}$-direction.

\section{E. Signal Pre-Processing \& Image Reconstruction}

We used the same signal pre-processing steps in both the simulations and experiments. First, the received signal was digitally high-pass filtered to remove any remaining direct feedthrough of the drive field. Next, for only experiments, a low-pass filter was applied to filter out the signal near and after the self-resonance frequency of the receive coil, which was measured at around $280 \mathrm{kHz}$. The resulting signal was further filtered for the purposes of spectrum cleaning. For the linear trajectories used in this work, we defined pass-bands around the harmonics, with a bandwidth of $420 \mathrm{~Hz}$ for simulations. Due to the relatively slower slew rate in the experiments, the signal spread to a narrow band around the harmonics. Therefore, a smaller pass-band bandwidth of $16 \mathrm{~Hz}$ was utilized for the experiments.

For PCI and Lumped-PCI, the pre-processed signal values were directly used to form the raw images, as described in Eq. 11a. Then, these images were interpolated to a finer grid of $0.05-\mathrm{mm}$ spacing and deconvolved by the kernels computed from Eq. 8 and Eq. 18, taking $\beta_{0}=1$. The deconvolution was performed using the built-in MATLAB function deconvreg, to reconstruct the final MPI images. Here, deconvreg implements a regularized filter algorithm that finds the least squares estimate under image smoothness constraint.

\section{F. Comparison of Image Quality}

Standard $\mathrm{x}$-space reconstruction with DC recovery algorithm [9] and SNR optimized pFOV stitching [24] was implemented for comparison purposes. The comparison and proposed techniques used identical signal pre-processing steps, as described in Section III-E.

For quantitative image quality assessment of the reconstructed images in simulations, the PSNR metric was employed:

$$
\operatorname{PSNR}(I)=10 \log _{10}\left(\frac{R^{2}}{M S E}\right)
$$

where

$$
M S E=\frac{\sum_{M, N}\left(I[m, n]-I_{r e f}[m, n]\right)^{2}}{M N}
$$

Here, $I[m, n]$ is the reconstructed image, $\mathrm{R}$ is the peak value that a pixel can have, $I_{r e f}[m, n]$ is the reference image, MSE is the mean-squared-error between the reconstructed image and the reference image, and images are of size $M \times N$. The phantom itself was used as the reference image, and all reconstructed images were individually normalized to [0 1] range. Accordingly, higher PSNR values indicate higher fidelity image reconstruction. 


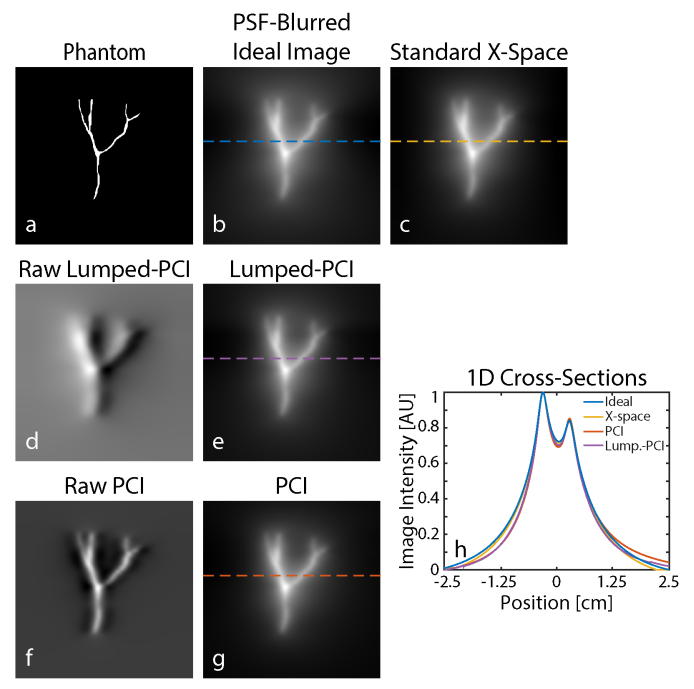

Fig. 4. Simulation results for ideal signal conditions. (a) A $5 \times 5 \mathrm{~cm}^{2}$ vasculature phantom and (b) the corresponding PSF-blurred ideal MPI image. Images reconstructed using (c) standard $x$-space reconstruction, (e) Lumped- $\mathrm{PCl}$, and (g) PCl. Here, (d) and (f) show the raw images for Lumped-PCl and $\mathrm{PCl}$, respectively. (h) $1 \mathrm{D}$ cross-sections of the reconstructed images. Under ideal signal conditions, all three methods provide visually similar results.

\section{Results}

\section{A. Simulation Results}

Figure 4 shows the results of the proposed and comparison reconstructions for a $5 \times 5 \mathrm{~cm}^{2}$ vasculature phantom for the ideal case, where noise, interference, and relaxation effects are neglected. Figure $4 a-4 c$ show the phantom, the PSF-blurred ideal MPI image, and the corresponding standard $\mathrm{x}$-space reconstruction result. Figure $4 \mathrm{~d}-4 \mathrm{~g}$ show the results of Lumped-PCI and PCI reconstructions together with the corresponding raw images. In addition, Fig. 4h shows 1D cross-sections of the reconstructed images to facilitate visual comparison. For this ideal case, all three methods yield visually similar images, particularly in the central regions of the FOV. A quantitative comparison of these images yields $13.4 \mathrm{~dB}, 13.5 \mathrm{~dB}$, and 14.1 dB PSNR values for standard $\mathrm{X}$-space, Lumped-PCI, and PCI, respectively. Although there is no visible difference between the reconstructed images, the PSNR values suggest that the PCI method is the most successful reconstruction method under ideal signal conditions.

Figure 5 shows example results for the proposed and comparison reconstructions at 4 different SNR levels between 10-40 dB. Both the standard $\mathrm{x}$-space and Lumped-PCI methods display robustness against noise. For standard $\mathrm{x}$-space, horizontal stripe artifacts appear for the lowest SNR level of $10 \mathrm{~dB}$, stemming from the inconsistencies during the stitching operation for different lines in the image. In contrast, an overall blurring is visible at the same SNR level for Lumped-PCI, due to the trade-off between noise regularization and resolution. The PCI method, on the other hand, shows degradation in image quality for the lowest two SNR levels. This relative noise sensitivity is expected, as PCI uses only a small portion of the received signal.

Figure 6 shows example results at 4 different SIR levels between 4-16 dB. Standard x-space reconstruction displays

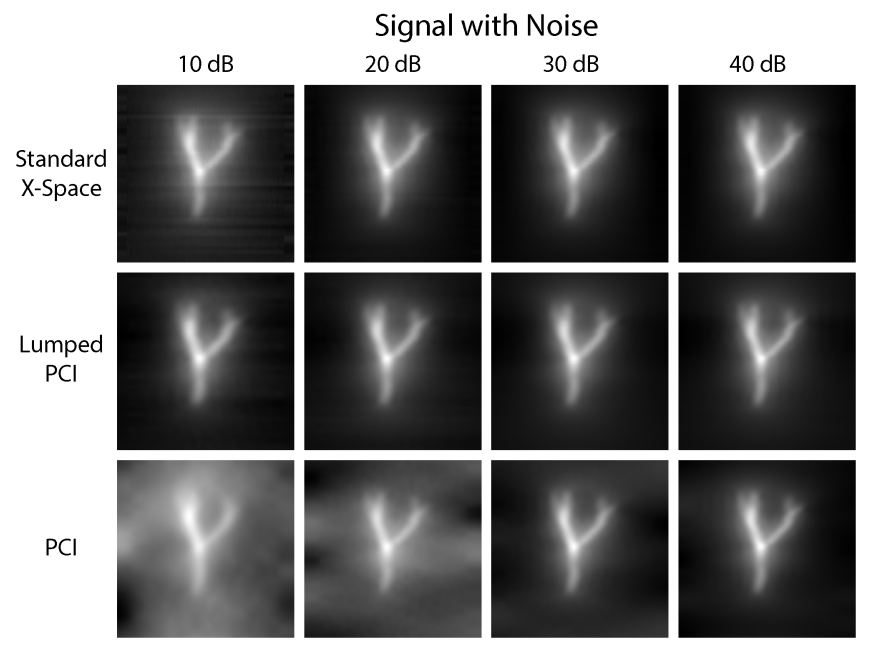

Fig. 5. Example results at 4 different SNR levels between 10-40 dB. Both standard $\mathrm{x}$-space and Lumped-PCI methods display robustness against noise, with slight horizontal stripe artifacts in standard $\mathrm{x}$-space and an overall blurring in Lumped-PCI at very low SNR levels. $\mathrm{PCI}$ shows degradation in image quality for the lowest two SNR levels, as it uses only a small portion of the received signal.

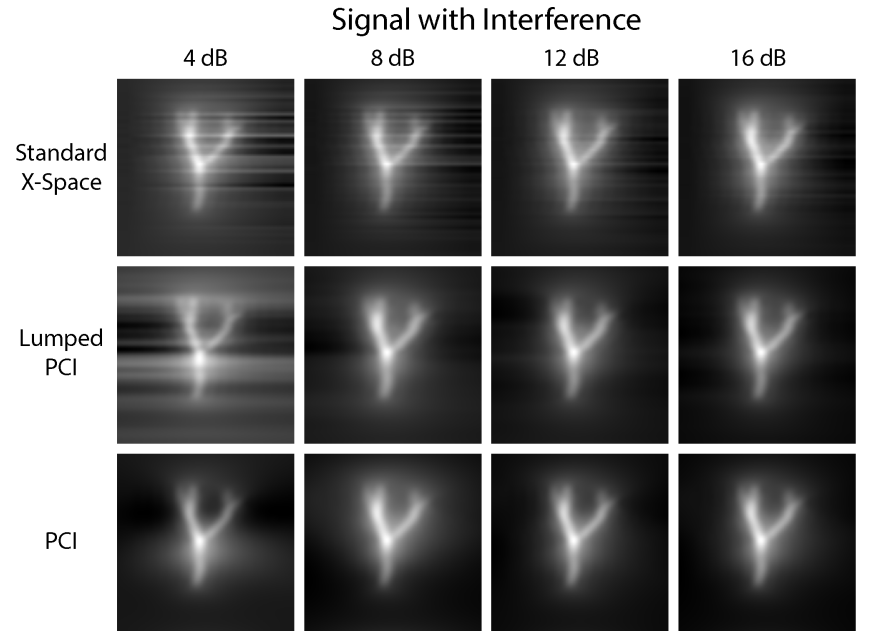

Fig. 6. Example results at 4 different SIR levels between 4-16 dB. Standard $\mathrm{x}$-space reconstruction suffers from horizontal stripe artifacts that are visible even at the highest SIR level of $16 \mathrm{~dB}$. Lumped-PCI is more robust against interference effects, however, thicker horizontal stripe artifacts arise at the lowest SIR level of $4 \mathrm{~dB}$. PCI demonstrates robustness against interference at all SIR levels.

sensitivity against harmonic interference, manifested as horizontal stripe artifacts that are visible even at the highest SIR level of $16 \mathrm{~dB}$. While Lumped-PCI is considerably more robust against interference effects, similar but thicker horizontal stripes emerge at the lowest SIR level of $4 \mathrm{~dB}$. In contrast, PCI demonstrates robustness against interference at all SIR levels tested. At the lowest SIR level, while there are image intensity differences in the background, these low-resolution features do not hinder the delineation of the phantom.

Figure 7 displays the combined effects of noise and harmonic interference on the three reconstruction methods, at a fixed SIR level of $8 \mathrm{~dB}$ with SNR ranging between 10-40 dB. The presence of harmonic interference limits the performance of the standard $\mathrm{x}$-space method, which suffers from stripe artifacts even at the highest SNR level of $40 \mathrm{~dB}$. Lumped-PCI 


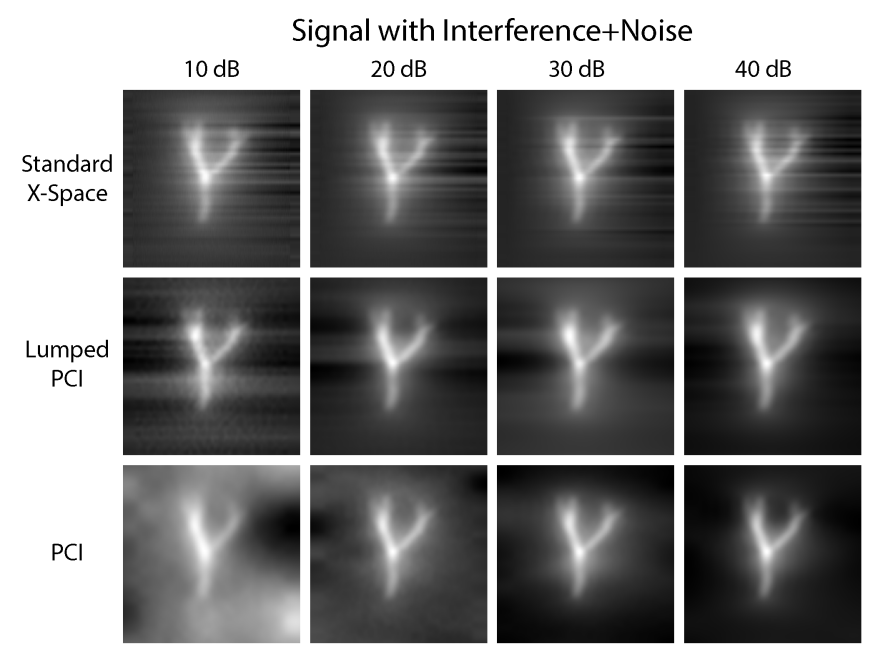

Fig. 7. Example results at 4 different SNR levels between 10-40 dB, with SIR fixed at $8 \mathrm{~dB}$. Due to harmonic interference, the standard $x$-space method exhibits stripe artifacts even at the highest SNR level of $40 \mathrm{~dB}$. Lumped-PCl shows improved image quality at all SNR levels, with relatively better behaved artifacts. $\mathrm{PCl}$ shows almost no artifacts at the highest two SNR levels, whereas its image quality degrades for the lowest two SNR levels.

shows improved image quality at that SNR level, with relatively better behaved artifacts at lower SNR levels. In contrast, PCI shows almost no artifacts at the highest two SNR levels. However, its image quality degrades considerably for the lowest two SNR levels. These results are in line with those in Fig. 5 and Fig. 6. When noise is the dominant effect, Lumped-PCI performs the best, whereas when interference dominates over noise, PCI yields the highest image quality.

Next, the image qualities of the three methods were compared quantitatively using the PSNR metric. At each SNR and SIR level, Monte Carlo simulations were performed by repeating the simulations 50 times, and the resulting PSNR values were averaged across repeats. The contour plots of the mean PSNR values are shown in Fig. 8, where the individual effects of noise and harmonic interference are also provided (see $\mathrm{SIR}=\infty$ and $\mathrm{SNR}=\infty$ levels, respectively). According to this figure, the main factor that determines the performance of standard $\mathrm{x}$-space is the interference level and not noise. Only for SNR $<20 \mathrm{~dB}$, the performance depends jointly on the noise and interference levels. While the performance trends for Lumped-PCI are similar, it outperforms standard $x$-space at all SNR and SIR levels. The PSNR difference between Lumped-PCI and standard $\mathrm{x}$-space increases as SNR and SIR decreases, e.g., the difference reaches $1.7 \mathrm{~dB}$ at $\mathrm{SNR}=10 \mathrm{~dB}$ and $\mathrm{SIR}=4 \mathrm{~dB}$. As expected, with its robustness against interference, PCI outperforms standard $\mathrm{x}$-space at moderate-to-high SNR levels (for SNR $>25 \mathrm{~dB}$ ). PCI also outperforms Lumped-PCI when SNR $>30 \mathrm{~dB}$. For example, at $\mathrm{SNR}=35 \mathrm{~dB}$ and $\mathrm{SIR}=8 \mathrm{~dB}$, the PSNR values are $10.6 \mathrm{~dB}, 10.9 \mathrm{~dB}$, and $12.0 \mathrm{~dB}$ for standard $\mathrm{x}$-space, LumpedPCI, and PCI, respectively. At low SNR levels, however, the performance of PCI quickly degrades, as noise effects dominate over interference.

Figure 9 displays the results of the proposed and comparison reconstructions for the combined effects of noise, interference, and relaxation. To investigate the effects of
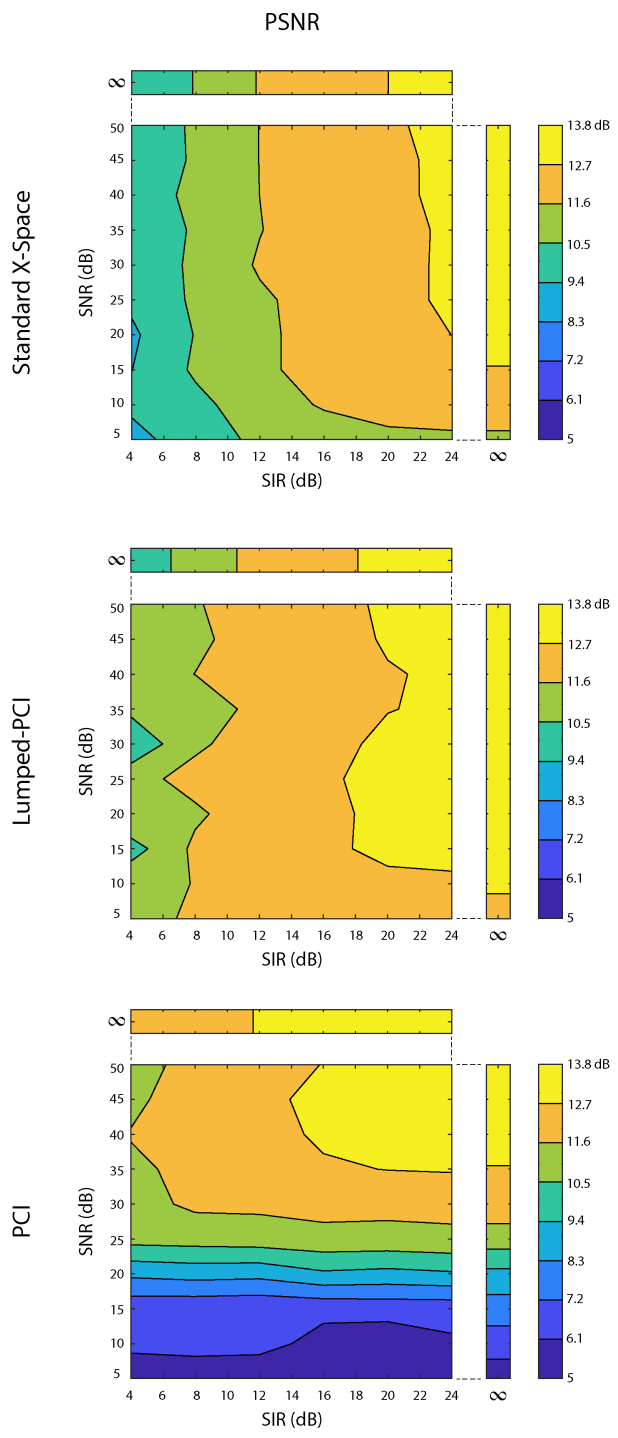

Fig. 8. PSNR contour plots showing image quality as a function of SNR and SIR. The individual effects of noise and harmonic interference can be seen at $\operatorname{SIR}=\infty$ and $\mathrm{SNR}=\infty$ levels, respectively. The performance of standard $x$-space is mainly dependent on the interference level and not noise, except for SNR $<20 \mathrm{~dB}$. Lumped-PCl outperforms standard $x$-space at all SNR and SIR levels. PCl yields the highest image quality when interference dominates over noise. $\mathrm{PCl}$ outperforms standard $x$-space when SNR $>25 \mathrm{~dB}$, and Lumped-PCl when SNR $>30 \mathrm{~dB}$.

relaxation, we assumed a realistic relaxation time constant of $\tau=3 \mu s$ [28]. For this analysis, SNR was fixed at $30 \mathrm{~dB}$ and SIR at $8 \mathrm{~dB}$. Comparing Fig. 9 with the third column of Fig. 7, one can see that relaxation causes a slight blurring for all three methods. The overall effects of noise and interference, however, remain the same as before. Once again, horizontal stripe artifacts are seen in the standard $\mathrm{x}$-space image. Both Lumped-PCI and PCI provide improved image quality with respect to standard $\mathrm{x}$-space. Under these specific conditions, PCI outperforms the other two methods. The PSNR values corresponding to standard $\mathrm{x}$-space, Lumped-PCI, and PCI are $10.6 \mathrm{~dB}, 11.9 \mathrm{~dB}$, and $12.6 \mathrm{~dB}$, respectively.

\section{B. Imaging Experiment Results}

Figure 10 displays the imaging experiment results of the proposed and comparison reconstructions using a phantom that 
Interference+Noise+Relaxation

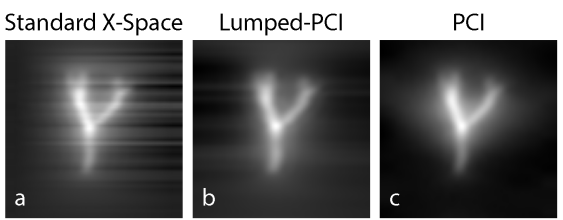

Fig. 9. Simulation results for a realistic scenario where noise, harmonic interference, and relaxation effects are all incorporated: $30 \mathrm{~dB}$ SNR, $8 \mathrm{db}$ SIR, relaxation time constant of $\tau=3 \mu \mathrm{s}$. The MPI images reconstructed by (a) standard $\mathrm{x}$-space, (b) Lumped- $\mathrm{PCl}$, and (c) $\mathrm{PCl}$ exhibit a slight blurring due to relaxation, but the overall effects of noise and interference remain the same as in Fig. 7. Under these conditions, $\mathrm{PCl}$ provides the best image quality, whereas Lumped-PCl also shows improved quality when compared to standard $\mathrm{x}$-space.

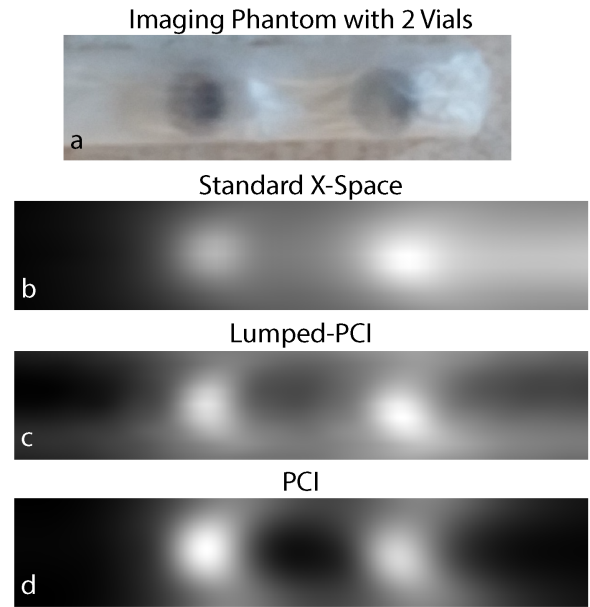

Fig. 10. Experimental imaging results using (a) an imaging phantom with two vials filled with identical concentration of Perimag nanoparticles, separated at 9-mm distance. The reconstructed MPI images from (b) standard x-space, (c) Lumped-PCl, and (d) PCl. Standard x-space suffers from a pile-up artifact in image intensity due to non-ideal signal conditions, whereas Lumped-PCl provides improved image quality with similar but less severe artifacts along the horizontal direction. $\mathrm{PCl}$ does not exhibit any artifacts and provides the highest image quality out of the three methods. FOV size: $0.7 \times 7.52 \mathrm{~cm}^{2}$, displayed FOV size: $0.7 \times 4.7 \mathrm{~cm}^{2}$

contains two vials of Perimag nanoparticles separated by a 9-mm distance. Standard $\mathrm{x}$-space suffers from a pile-up artifact in image intensity due to non-ideal signal conditions. Here, imaging experiments utilized 9 lines to cover the 2D FOV, as opposed to 51 lines used in the simulations. Therefore, the visual manifestation of the aforementioned horizontal stripe artifact is now a more dominant pile-up artifact. While Lumped-PCI also demonstrates similar but less severe artifacts along the horizontal direction, the image quality is visibly improved when compared to standard $\mathrm{x}$-space. On the other hand, PCI does not exhibit any artifacts and provides the highest image quality out of the three methods. Note that despite containing identical concentrations and volumes of the same nanoparticle, there is a visible intensity difference between the two vials for PCI, which potentially stems from an imperfect alignment of the phantom plane with respect to the 2D imaging plane. In standard $\mathrm{x}$-space, the intensity difference is reversed because of the dominant pile-up artifact.

The experimental results in Fig. 11 show that the proposed methods can handle different nanoparticle types. Here, three

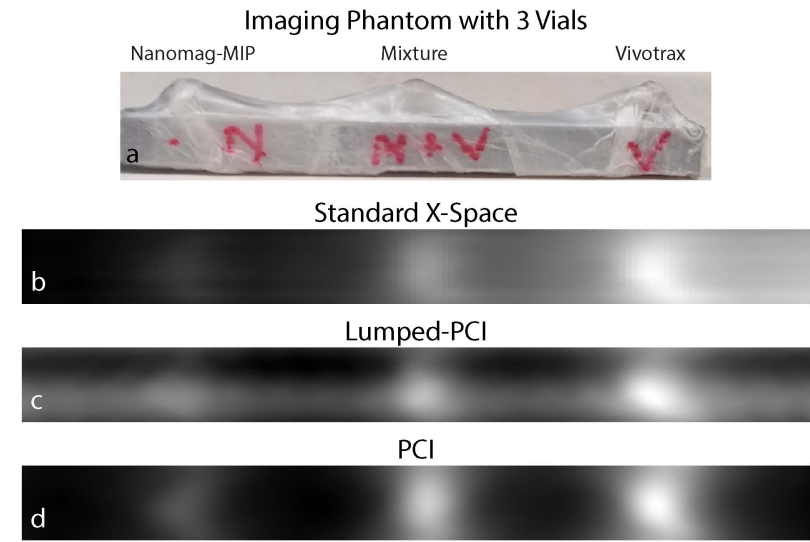

Fig. 11. Experimental imaging results using different types of nanoparticles. (a) The imaging phantom of three vials filled with Nanomag-MIP, Vivotrax, and a homogeneous mixture of the two. The reconstructed MPI images from (b) standard x-space, (c) Lumped-PCl, and (d) PCl. $\mathrm{PCl}$ gives the highest image quality out of the three methods. The results show that the proposed methods can successfully handle different nanoparticle types. FOV size: $0.7 \times 7.52 \mathrm{~cm}^{2}$.

vials filled with Nanomag-MIP, VivoTrax, and a homogeneous mixture of the two separated by $15-\mathrm{mm}$ distances were imaged. Once again, standard $\mathrm{x}$-space suffers from an intensity pile-up on the right hand side of the image. While the image quality of Lumped-PCI is improved with respect to standard $\mathrm{x}$-space, the effects of interference can still be observed in the horizontal direction. PCI is free from such artifacts and provides the highest image quality due to its robustness against harmonic interference effects.

For the experiments in Figs. 10 and 11, the SNR and SIR levels were computed from the MPI signals in time domain and frequency domain, respectively. These computations yielded $\mathrm{SNR}=32.3 \mathrm{~dB}$ and $\mathrm{SIR}=7.9 \mathrm{~dB}$ for the experiments in Fig. 10, and SNR $=31.1 \mathrm{~dB}$ and SIR $=4.8$ $\mathrm{dB}$ for those in Fig. 11. The parameters for the simulations in Fig. 9 were based on these experimental results. One can see that the overall effects of non-ideal signal conditions in the experiments are consistent with those seen in the simulations. These proof-of-concept experiments demonstrate both the feasibility with different nanoparticle types and robustness against non-ideal signal conditions for the proposed methods.

\section{DISCUSSION}

In this work, with simulations and experimental results, we have shown that PCI provides improved robustness against harmonic interferences when compared to standard $\mathrm{x}$-space reconstruction. In cases where using only a small portion of the received signal renders PCI sensitive to noise, we propose to improve its noise robustness by lumping the signals from the entire pFOV. Utilizing the edges of the pFOVs reduces interference robustness of Lumped-PCI, hence, a clear trade-off between noise robustness and interference robustness emerges. To adjust to a specific SNR and SIR level, a central region of pFOV can be lumped instead of the entire pFOV. For example, the signals at $\mathrm{pFOV}$ edges can be discarded without significant data loss to improve interference robustness. Similarly, standard $\mathrm{x}$-space reconstruction also proposed using the central 
$95 \%$ of pFOV to avoid velocity-compensation-induced noise amplification at the edges [9]. Note that while this procedure improves noise robustness of $\mathrm{x}$-space reconstruction, it does not provide sufficient robustness against interference. In fact, the experimental results in Fig. 10b and Fig. 11b utilized the central $90 \%$ of $\mathrm{pFOV}$ for $\mathrm{x}$-space reconstruction, but still exhibited pile-up artifacts.

There are multiple potential sources of harmonic interferences in MPI, most notably the non-linearities in the transmit chain (e.g., from power amplifier or capacitors). Especially if the active/passive compensation of the direct feedthrough does not provide sufficient decoupling between the transmit and receive coils, the higher harmonics of the drive field can also feed through to the MPI signal. In addition, insufficient shielding may cause eddy currents on the selection field permanent magnets, which in turn can induce signal on the receive coil [32], [33]. Likewise, ambient interferences may also become problematic due to insufficient shielding of the receive coil and/or transmit/receive filter chains [34]. Under low signal conditions, such as in vivo cases, these harmonic interferences can become a problem even for well-tuned systems. Therefore, the reconstruction technique proposed in this work can improve image quality not just for low-cost MPI scanners (e.g., like the FFP scanner used in this work), but also for high-fidelity commercial systems. Moreover, the proposed PCI method substantially simplifies the reconstruction procedure by eliminating the need for individual pFOV processing (i.e., gridding, DC shift calculation, and stitching).

It should be emphasized that while the proposed method is demonstrated for linear trajectories, it is applicable to any trajectory that contains a 1D drive field with a slowly varying focus field. The sizes of the kernels in Eqs. 8 and 18 become more compact at smaller drive field amplitudes, which would yield a higher fidelity deconvolution with reduced noise-amplification effects. One of the aforementioned requirements of PCI is that the pFOV centers are closely spaced, such that there are multiple samples per kernel length $W$ (i.e., the pFOV extent). Note that this requirement can be thought to be identical to the overlapping $\mathrm{pFOV}$ requirement of standard $\mathrm{X}$-space reconstruction. Nevertheless, due to the $20 \mathrm{~T} / \mathrm{s}$ safety limit on the slew rate of the focus field [14], realistic scan trajectories must already consist of closely spaced pFOVs. For the experiments in this work, using a drive field at $9.7 \mathrm{kHz}$ with a slew rate of $70 \mathrm{mT} / \mathrm{s}$ yielded $3-\mu \mathrm{m}$ distances between pFOV centers. This level of spacing is more than sufficient to reconstruct a high quality image, as it is three orders of magnitude below the expected mm-range resolution of our scanner and is well below typical pFOV sizes. When operating at the safety limit of $20 \mathrm{~T} / \mathrm{s}$ slew rate [14], the distances between pFOV centers would scale up to $0.86 \mathrm{~mm}$. While this spacing may also be sufficient in most cases, the slew rate of the focus field can be reduced accordingly to attain a desired spacing level. Demonstration of PCI at such high slew rates remains as future work, as it requires incorporating electromagnetically driven focus fields.

The compact kernels used in PCI and Lumped-PCI both have fully-known shapes that solely depend on the pFOV size. The scaling factor $\beta_{0}$, however, depends on the nanoparticle type. Using a constant $\beta_{0}$ (as done in this work) results in a global scaling of the reconstructed image for cases with a single type of nanoparticle, and nanoparticle-dependent scaling of pixel intensities for cases with more than one type of nanoparticle. It is important to note that an identical scaling effect is also present in standard $\mathrm{x}$-space reconstruction, as the speed compensation step does not correct for the nanoparticle-dependent parameter $\alpha$ in Eq. 1. Nevertheless, in the case of single type of nanoparticle, the benign global scaling can easily be corrected by imaging a source with known concentration and rescaling other images respectively. For cases with more than one type of nanoparticle, one potential solution to correct for nanoparticle-dependent scaling is to measure the relative values of $\beta_{0}$ by performing a calibration scan using point sources of identical concentrations. Note that this measurement could also be performed on a magnetic particle spectrometer (MPS) setup, as the relative scaling of $\beta_{0}$ values is independent of the system parameters. One can then determine the spatial distribution of each nanoparticle type using multi-color MPI techniques [28], [35], and achieve true pixel intensities via local rescaling of the reconstructed image by the relative $\beta_{0}$ values. Demonstration of this approach remains an important future extension of the proposed PCI method.

The proposed method also has the potential to be combined with some of the recently proposed $\mathrm{x}$-space reconstruction approaches. A recent work has shown that one can achieve isotropic resolution with standard $\mathrm{x}$-space reconstruction by combining images from two orthogonal scanning directions [36]. The resolution of the resulting isotropic image can then be further improved by using an equalization filter that extracts the image component stemming from the narrower tangential PSF [18], [37]. By replacing the standard X-space reconstruction step with PCI, the harmonic interference robustness of these multi-channel acquisition and equalization approaches can be significantly improved. Another recent work proposed a hybrid method that combines an x-space-based reconstruction with a system function approach [38]. This method first uses a gridding based reconstruction to generate a raw image, followed by an image-based system matrix reconstruction to correct for system-induced geometric warping and position-dependent blurring effects. Likewise, a hybrid extension of PCI that incorporates system function reconstruction could also be pursued to gain robustness against magnetic field inhomogeneities of an MPI scanner, along with the robustness against harmonic interferences.

\section{CONCLUSION}

In this work, we have proposed a robust $\mathrm{x}$-space image reconstruction that consists of two simplified steps: forming a raw image by directly assigning the signal to pFOV center locations, and deconvolving the raw image by a known, narrow kernel. Extensive simulation results and imaging experiments demonstrated that the proposed PCI method outperforms standard $\mathrm{x}$-space reconstruction. PCI is particularly robust against harmonic interferences, making it a promising technique for in vivo applications. In SNR starved cases, the noise robustness of PCI can be further improved by lumping the signals from the 
entire pFOV, with a trade-off of slightly reduced interference robustness. The proposed method promises a computationally simpler and straightforward reconstruction with high-fidelity image reconstruction.

\section{ACKNOWLEDGMENT}

The authors would like to thank O. B. Demirel for his contributions to the MPI simulation toolbox, and M. Utkur for his assistance in the experimental setup.

\section{References}

[1] B. Gleich and J. Weizenecker, "Tomographic imaging using the nonlinear response of magnetic particles," Nature, vol. 435, no. 7046, pp. 1214-1217, Jun. 2005.

[2] J. Weizenecker, J. Borgert, and B. Gleich, "A simulation study on the resolution and sensitivity of magnetic particle imaging," Phys. Med. Biol., vol. 52, no. 21, pp. 6363-6374, Nov. 2007.

[3] P. W. Goodwill et al., "X-space MPI: Magnetic nanoparticles for safe medical imaging," Adv. Mater., vol. 24, no. 28, pp. 3870-3877, Jul. 2012.

[4] E. U. Saritas et al., "Magnetic particle imaging (MPI) for NMR and MRI researchers," J. Magn. Reson., vol. 229, pp. 116-126, Apr. 2013.

[5] L. M. Bauer, S. F. Situ, M. A. Griswold, and A. C. S. Samia, "Magnetic particle imaging tracers: State-of-the-art and future directions," J. Phys. Chem. Lett., vol. 6, no. 13, pp. 2509-2517, Jul. 2015.

[6] B. Zheng et al., "Seeing SPIOs directly in vivo with magnetic particle imaging," Mol. Imag. Biol., vol. 19, no. 3, pp. 385-390, Jun. 2017.

[7] E. U. Saritas, P. W. Goodwill, G. Z. Zhang, and S. M. Conolly, "Magnetostimulation limits in magnetic particle imaging," IEEE Trans. Med. Imag., vol. 32, no. 9, pp. 1600-1610, Sep. 2013.

[8] I. Schmale, B. Gleich, J. Rahmer, C. Bontus, J. Schmidt, and J. Borgert, "MPI safety in the view of MRI safety standards," IEEE Trans. Magn. vol. 51, no. 2, pp. 1-4, Feb. 2015

[9] K. Lu, P. W. Goodwill, E. U. Saritas, B. Zheng, and S. M. Conolly, "Linearity and shift invariance for quantitative magnetic particle imaging," IEEE Trans. Med. Imag., vol. 32, no. 9, pp. 1565-1575, Sep. 2013.

[10] M. Gruttner, T. F. Sattel, F. Griese, and T. M. Buzug, "System matrices for field of view patches in magnetic particle imaging," Proc. SPIE, vol. 8672, Mar. 2013, Art. no. 86721A.

[11] P. Szwargulski, M. Ahlborg, C. Kaethner, and T. M. Buzug, "Trajectory analysis using static patches for magnetic particle imaging," IEEE Trans. Magn., vol. 51, no. 2, pp. 1-4, Feb. 2015.

[12] T. Knopp and M. Kaul, "MPI focus field experiments using non-overlapping focus-field patches," in Proc. 5th Int. Workshop Magn. Part. Imag. (IWMPI), Mar. 2015, p. 1.

[13] J. J. Konkle, P. W. Goodwill, E. U. Saritas, B. Zheng, K. Lu, and S. M. Conolly, "Twenty-fold acceleration of 3D projection reconstruction MPI," Biomedizinische Technik/Biomed. Eng., vol. 58, no. 6, pp. 565-576, Jan. 2013.

[14] International Commission on Non-Ionizing Radiation Protection, "Medical magnetic resonance (MR) procedures: Protection of patients," Health Phys., vol. 87, no. 2, pp. 197-216, 2004.

[15] P. W. Goodwill and S. M. Conolly, "The X-space formulation of the magnetic particle imaging process: 1-D signal, resolution, bandwidth, SNR, SAR, and magnetostimulation," IEEE Trans. Med. Imag., vol. 29, no. 11, pp. 1851-1859, Nov. 2010

[16] P. W. Goodwill and S. M. Conolly, "Multidimensional X-space magnetic particle imaging," IEEE Trans. Med. Imag., vol. 30, no. 9 , pp. 1581-1590, Sep. 2011.

[17] J. J. Konkle, P. W. Goodwill, D. W. Hensley, R. D. Orendorff, M. Lustig, and S. M. Conolly, "A convex formulation for magnetic particle imaging X-space reconstruction," PLoS ONE, vol. 10, no. 10, Oct. 2015 , Art. no. e0140137.
[18] A. A. Ozaslan, A. Alacaoglu, O. B. Demirel, T. Çukur, and E. U. Saritas, "Fully automated gridding reconstruction for non-Cartesian X-space magnetic particle imaging," Phys. Med. Biol., vol. 64, no. 16, Aug. 2019, Art. no. 165018

[19] Z. W. Tay et al., "Pulsed excitation in magnetic particle imaging," IEEE Trans. Med. Imag., vol. 38, no. 10, pp. 2389-2399, Oct. 2019.

[20] M. Utkur and E. U. Saritas, "Comparison of different coil topologies for an MPI relaxometer," in Proc. 5th Int. Workshop Magn. Part. Imag. (IWMPI), Mar. 2015, p. 1.

[21] P. W. Goodwill, J. J. Konkle, B. Zheng, E. U. Saritas, and S. T. Conolly, "Projection X-space magnetic particle imaging," IEEE Trans. Med. Imag., vol. 31, no. 5, pp. 1076-1085, May 2012.

[22] M. Graeser, T. Knopp, M. Grüttner, T. F. Sattel, and T. M. Buzug, "Analog receive signal processing for magnetic particle imaging," Med. Phys., vol. 40, no. 4, Apr. 2013, Art. no. 042303.

[23] D. Pantke, N. Holle, A. Mogarkar, M. Straub, and V. Schulz, "Multifrequency magnetic particle imaging enabled by a combined passive and active drive field feed-through compensation approach," Med. Phys., vol. 46, no. 9, pp. 4077-4086, Sep. 2019.

[24] E. Bozkurt and E. U. Saritas, "Signal-to-noise ratio optimized image reconstruction technique for magnetic particle imaging," J. Fac. Eng. Archit. Gazi Univ., vol. 32, no. 3, pp. 999-1013, 2017.

[25] J. Rahmer, J. Weizenecker, B. Gleich, and J. Borgert, "Signal encoding in magnetic particle imaging: Properties of the system function," $B M C$ Med. Imag., vol. 9, no. 1, p. 4, Dec. 2009.

[26] D. Sarica, O. B. Demirel, and E. U. Saritas, "DC shift based image reconstruction for magnetic particle imaging," in Proc. 25th Signal Process. Commun. Appl. Conf. (SIU), May 2017, pp. 1-4.

[27] S. Kurt, Y. Muslu, M. Utkur, and E. U. Saritas, "Harmonic dispersion X-space MPI,' in Proc. 9th Int. Workshop Magn. Part. Imag. (IWMPI), Mar. 2019, pp. 75-76.

[28] Y. Muslu, M. Utkur, O. B. Demirel, and E. U. Saritas, "Calibration-free relaxation-based multi-color magnetic particle imaging," IEEE Trans. Med. Imag., vol. 37, no. 8, pp. 1920-1931, Aug. 2018.

[29] M. Utkur, Y. Muslu, and E. U. Saritas, "Relaxation-based color magnetic particle imaging for viscosity mapping," Appl. Phys. Lett., vol. 115, no. 15 , Oct. 2019, Art. no. 152403.

[30] L. R. Croft, P. W. Goodwill, and S. M. Conolly, "Relaxation in X-space magnetic particle imaging," IEEE Trans. Med. Imag., vol. 31, no. 12, pp. 2335-2342, Dec. 2012.

[31] M. Utkur, Y. Muslu, and E. U. Saritas, "A 4.8 T/m magnetic particle imaging scanner design and construction," in Proc. 21st Nat. Biomed. Eng. Meeting (BIYOMUT), Nov./Dec. 2017, pp. 1-4.

[32] P. W. Goodwill, K. Lu, B. Zheng, and S. M. Conolly, "An X-space magnetic particle imaging scanner," Rev. Sci. Instrum., vol. 83, no. 3, Mar. 2012, Art. no. 033708.

[33] D. M. Yalcinkaya, M. Utkur, and E. U. Saritas, "Finite element analysis of passive magnetic shields for a FFP MPI scanner," in Proc. 8th Int. Workshop Magn. Part. Imag. (IWMPI), Mar. 2018, pp. 123-124.

[34] L. M. Bauer et al., "Eddy current-shielded X-space relaxometer for sensitive magnetic nanoparticle characterization," Rev. Sci. Instrum., vol. 87, no. 5, May 2016, Art. no. 055109.

[35] J. Rahmer, A. Halkola, B. Gleich, I. Schmale, and J. Borgert, "First experimental evidence of the feasibility of multi-color magnetic particle imaging," Phys. Med. Biol., vol. 60, no. 5, pp. 1775-1791, Mar. 2015.

[36] K. Lu, P. Goodwill, B. Zheng, and S. Conolly, "Multi-channel acquisition for isotropic resolution in magnetic particle imaging," IEEE Trans. Med. Imag., vol. 37, no. 9, pp. 1989-1998, Sep. 2018.

[37] K. Lu, P. Goodwill, B. Zheng, and S. Conolly, "Reshaping the 2D MPI PSF to be isotropic and sharp using vector acquisition and equalization," in Proc. 5th Int. Workshop Magn. Part. Imag. (IWMPI), Mar. 2015, p. 1

[38] P. Vogel, T. Kampf, M. A. Rückert, and V. C. Behr, "Flexible and dynamic patch reconstruction for traveling wave magnetic particle imaging," Int. J. Magn. Part. Imag., vol. 2, no. 2, 2016, Art. no. 611001 